\title{
Editorial:
}

\section{La Inclusión como Dilema}

Cynthia Duk * 1

F. Javier Murillo ${ }^{2}$

La inclusión en el campo de la educación es esencialmente compleja de abordar en términos de los diversos significados que se le atribuyen en distintos contextos y de la amplitud que ha ido adquiriendo como resultado de su evolución. De esta forma no sería muy arriesgado afirmar que el concepto de inclusión educativa tiene límites poco nítidos, es a veces ambiguo y otras controversial y dilemático, se ha ido desarrollando gracias a un proceso de extensión progresiva, incorporándose como aspiración en la agenda pública a nivel internacional y de numerosos países incidiendo crecientemente en las decisiones de política en los sistemas educativos y sus instituciones. Si bien es difícil determinar la procedencia de este término y el aumento que ha experimentado su uso durante las últimas décadas -su expansión data de mediados de los 90-, lo cierto es que en la actualidad es un término obligado en los documentos y discursos políticos, convirtiéndose incluso en una especie de "slogan" dentro de lo que cabría llamar un pensamiento política y éticamente adecuado. Algunos expertos y seguidores críticos del movimiento de inclusión han puesto de manifiesto con cierta preocupación que este término se ha transformado en una "moda internacional", carente muchas veces de sentidos y coherencia.

Todo ello nos lleva a defender la importancia de construir una visión compartida acerca de qué es y qué significa a inclusión en educación que nos permita una comprensión más profunda de su significado y sus alcances desde la complejidad que involucra la mirada sistémica y muldimensional que una perspectiva global de la misma requiere. Lo anterior con la finalidad última de que nos sirva en mayor medida para orientar las decisiones educativas y su puesta en práctica.

Como punto de partida, es fundamental no perder de vista que la inclusión, en tanto componente del derecho a la educación, es un principio rector que debe orientar el curso de las políticas y prácticas educativas, teniendo como eje fundamental el reconocimiento y valoración de la diversidad humana. La UNESCO (2005) la define como un medio para lograr el acceso equitativo de la diversidad del alumnado a una educación de calidad, sin ningún tipo de discriminación. La inclusión como derecho exige que todas las escuelas acojan a los niños, niñas y jóvenes de la comunidad independiente de sus capacidades, origen sociocultural, genero, etnia, orientación sexual, situaciones de vida.

En este marco, algunos de los elementos recurrentes en la literatura que definen y dan sentido a la inclusión son los siguientes:

- La inclusión tiene que ver con todos los estudiantes y no solo con aquellos tradicionalmente considerados "diferentes" "especiales" o "vulnerables".

*Contacto: cduk@ucentral.cl

ISSN: 0718-7378

www.rinace.net/rlei/ 
- La inclusión busca maximizar la presencia, la participación y el aprendizaje de todos los y las estudiantes y, por tanto, supone identificar y remover las barreras de distinta índole que limitan sus oportunidades educativas.

- La inclusión pone particular énfasis en transformar los mecanismos que excluyen y discriminan a las y los estudiantes, implica el reconocimiento y valoración de las distintas culturas, identidades, características y capacidades de todos los estudiantes.

- La inclusión es un proceso nunca acabado, supone una búsqueda constante de mejoras e innovaciones para responder más positivamente a la diversidad del alumnado y, en consecuencia, implica un proceso continuo de aprendizaje y desarrollo en el conjunto del sistema educativo.

Igual de importante que contar con una definición clara, es comprender la naturaleza intrínseca del proceso de inclusión que como bien afirman los profesores Echeita, Simón, Sandoval y López (2011), se trata de un proceso esencialmente dilemático. En palabras de estos autores no habría "una política o una práctica educativa que pueda conseguir hoy por hoy el $100 \%$ de satisfacción en términos de asegurar la plena presencia, el óptimo aprendizaje y la participación completa de todos los estudiantes en los sistemas educativos" (Echeita et al., 2011, p. 3).

El dilema fundamental que se mueve alrededor de los sistemas generales de educación, dice relación con cómo lograr el difícil equilibrio ente lo común y lo diverso. Es decir el dilema de cómo ofrecer una educación pertinente, ajustada a las diferencias socioculturales e individuales de los y las estudiantes y, al mismo tiempo, hacerlo en el marco de estructuras comunes e inclusivas (sistema, escuela y aula), que garanticen condiciones de igualdad y favorezcan la convivencia en la diversidad, el sentido de pertenencia y la cohesión social.

Siguiendo a estos autores (Echeita et al., 2011) y recogiendo los aportes de Alan Dyson (2010), en nuestro intento por comprender mejor la noción de dilema desde la perspectiva de la inclusión, diríamos que es importante tener en consideración que:

- Los dilemas por definición, no se resuelven de manera definitiva. Se refieren a situaciones que involucran alternativas de acción contrarias, ninguna de las cuales es completamente deseable, poniendo al sujeto en el centro de la disyuntiva respecto de que opción escoger. Siempre hay elementos positivos y negativos en las opciones en juego, por lo mismo es un problema.

- Lo que cabe es resolverlos episódicamente, como resultado de un proceso de toma de decisiones en el que finalmente se toma aquella opción que tiene más elementos a favor que en contra, o porque se tiene en consideración aquella alternativa que tiene más peso, relevancia o impacto presente o futuro en la vida de los estudiantes.

- Por último, los dilemas educativos no se resuelven en el vacío, sino condicionados por una serie de factores en interacción que es necesario tener en cuenta.

Las concepciones o creencias implícitas de los actores educativos respecto de las opciones en conflicto y sus valores individuales. 
Los valores sociales y las ideologías mayoritarias presentes en el contexto en el que tienen lugar.

$\checkmark$ Las políticas educativas que las administraciones centrales o locales mantienen o promueven.

$\checkmark$ El grado de influencia o "poder" que puedan ostentar los actores que intervienen, ya sea debido a su estatus, antigüedad, cargo u otro.

$\checkmark$ Los recursos económicos, humanos, didácticos o tecnológicos que, en cada caso, puedan estar especialmente relacionados con la situación dilemática.

En definitiva, el carácter dilemático de la inclusión explica en buena parte su problemática y complejidad a la hora de la toma de decisiones en cualquiera de sus niveles, derivando en efectos más o menos deseables o favorables, dependiendo de las variables contextuales y culturales que entran en juego para su resolución. Podemos aventurar que también explicaría en parte, los desarrollos disímiles que se aprecian tanto en los planteamientos como en los procesos y prácticas de inclusión entre distintos sistemas educativos, comunidades escolares e incluso entre los actores educativos como individuos.

Para concluir presentamos a continuación tres dilemas relacionados con la inclusión que tienen incidencias en el reconocimiento, aprendizaje y participación de los estudiantes, que esperamos ayuden a ilustrar los planteamientos que hemos desarrollado en este texto. En el primero incluimos los argumentos de valor que pueden apoyar una u otra opción en conflicto. Los últimos dos ejemplos los dejamos abiertos a la reflexión.

\section{El dilema de la identificación}

Uno de los dilemas que suelen enfrentar las administraciones educativas, es la decisión de utilizar mecanismos de identificación y clasificación de los estudiantes en determinadas categorías, como por ejemplo alumnos "vulnerables", "con necesidades educativas especiales", "en riesgo de fracaso escolar", para asignar y distribuir de manera focalizada los recursos disponibles y proporcionarle a los grupos en situación de desventaja apoyos adicionales o programas diferenciados en función de sus particulares necesidades. Algunos de los argumentos a favor de estas medidas compensatorias, dicen relación con el uso más eficiente y regulado de los recursos siempre insuficientes y las ventajas de las estrategias de focalización para asegurar que los apoyos lleguen a los beneficiarios. Los argumentos en contra de esta opción, se basan en los efectos nocivos del uso de etiquetas, en particular cuando estas se asocian a atributos socialmente devaluados lo que no solo afecta el autoconcepto de los estudiantes, sino que además condiciona significativamente las expectativas de los profesores, los propios estudiantes y sus familias, con las consiguientes repercusiones en su desarrollo y aprendizaje.

En contraposición a esta alternativa, está la opción de que las escuelas dispongan de sistemas y recursos de apoyo que beneficien al conjunto de la comunidad escolar y a cualquier estudiante que los pueda requerir en algún momento de su trayectoria escolar, sin que estos se limiten a determinadas categorías de estudiantes. Contar con sistemas de apoyo que colaboren con el profesorado para abordar el desafío de una enseñanza más efectiva y adecuada a la diversidad del alumnado, es uno de los aspectos positivos y más promisorios de esta opción. Pero, al mismo tiempo, una excesiva flexibilidad en el uso y 
destinación de los recursos de apoyo en manos de las escuelas tiene el riesgo de que estos se diluyan entre las múltiples demandas que tienen las escuelas, o que se invisibilicen las necesidades de los estudiantes que requieren más ayuda.

\section{El dilema del agrupamiento}

En el caso de las escuelas, uno de los dilemas que a muchos directivos y docentes se les presenta, tiene relación con los criterios que deciden utilizar para distribuir a los estudiantes en los grupos. La disyuntiva es organizar los cursos y los agrupamientos al interior del aula, buscando que queden conformados de manera equilibrada en cuanto a la diversidad de características de los estudiantes, o conformarlos lo más homogéneamente posible, agrupando a los estudiantes en función de características y rendimiento académico similar.

\section{El dilema de la promoción}

Otro dilema al que a menudo se ven enfrentados especialmente los profesores, es al dilema de la promoción de curso o gado versus la repitencia. ¿Qué opción tomar frente a un estudiante que no ha alcanzado un rendimiento suficiente respecto de los aprendizajes esperados que establece el currículo, dejarlo repitiendo de curso, o pasarlo al nivel siguiente?, con las consecuencias que una u otra alternativa puede suponer para su desarrollo actual y futuro.

No sin justificación escuchamos una y otra vez que la educación inclusiva es uno de los mayores retos que hoy enfrentan los sistemas educativos. Los focos y perspectivas que demarcan la inclusión en los distintos países van variando según los avances y dificultades que emergen en cada contexto. Uno de esos retos, se vincula con lo que la investigación ha demostrado hace ya tiempo, la importancia de construir relaciones sinérgicas entre escuela, familia y comunidad, como uno de los factores clave para avanzar hacia sistemas y escuelas más inclusivas. Es precisamente esta temática la que aborda el presente número de nuestra revista, el cual ha sido coordinado por la Dra. Cecilia Simón como editora invitada, a quien agradecemos por la excelente calidad del trabajo realizado.

\section{Referencias}

Dyson, A. (2010) Cambios en la perspectiva sobre la educación especial desde un enfoque inglés. Revista Educación Inclusiva, 3(1), 69-84.

Echeita, G., Simón, C., Sandoval, M. y López, M. (2011). Módulo I: Inclusión educativa y diversidad. diplomado "escuelas inclusivas. enseñar y aprender en la diversidad". Madrid: OEI - Universidad Central de Chile.

UNESCO. (2005). Informe de seguimiento de la educación para todos en el mundo. El imperativo de la calidad. París: UNESCO 